\title{
An Absolute Standardized Uptake Value Is More Useful than the Decreased Rate of Uptake of FDG-PET to Predict Responses to Neoadjuvant Chemoradiotherapy for Esophageal Cancer
}

\author{
Masanobu Nakajima1, Hiroyuki Kato1, Tatsuya Miyazaki², Takanori Inose ${ }^{2}$, Naritaka Tanaka², \\ Shigemasa Suzuki², Takehiko Yokobori², Makoto Sakai², Akihiko Sano², Keisuke Ieta², \\ Makoto Sohda', Minoru Fukuchi², Hiroyuki Kuwano² \\ ${ }^{1}$ Department of Surgery I, Dokkyo Medical University, Mibu, Japan \\ ${ }^{2}$ Department of General Surgical Science, Gunma University Graduate School of Medicine, Maebashi, Japan \\ Email: mnakajim@dokkyomed.ac.jp
}

How to cite this paper: Nakajima, M., Kato, H., Miyazaki, T., Inose, T., Tanaka, N., Suzuki, S., Yokobori, T., Sakai, M., Sano, A., Ieta, K., Sohda, M., Fukuchi, M. and Kuwano, H. (2016) An Absolute Standardized Uptake Value Is More Useful than the Decreased Rate of Uptake of FDG-PET to Predict Responses to Neoadjuvant Chemoradiotherapy for Esophageal Cancer. Open Journal of Gastroenterology, 6, 373-385. http://dx.doi.org/10.4236/ojgas.2016.611040

Received: October 25, 2016

Accepted: November 27, 2016

Published: November 30, 2016

Copyright $\odot 2016$ by authors and Scientific Research Publishing Inc. This work is licensed under the Creative Commons Attribution International License (CC BY 4.0).

http://creativecommons.org/licenses/by/4.0/

\begin{abstract}
Background: Neoadjuvant chemoradiotherapy (CRT) is frequently performed for esophageal squamous cell carcinoma. In this study, we retrospectively assessed the standardized uptake value (SUV) of FDG-PET against decreased rates of SUV to assess the response of advanced esophageal squamous cell carcinoma patients to neoadjuvant CRT, and the correlation of this response with histopathological findings. Patients and Methods: Thirty-three patients receiving CRT followed by surgery were analyzed. Results: Using the decreased rate of maximum SUV, the sensitivity and specificity in distinguishing complete responders (CR) from non-CR patients was $63 \%$ and $44 \%$. Using the maximum SUV before surgery, the sensitivity and specificity for distinguishing pathological CR from non-CR was $88 \%$ and $56 \%$. Conclusions: To identify complete responders of CRT for esophageal cancer, absolute maximum SUV value is a better predictor than decreased rate of the maximum SUV.
\end{abstract}

\section{Keywords}

Esophageal Cancer, Neoadjuvant Therapy, Chemoradiotherapy, FDG-PET, SUV

\section{(c) (i) Open Access}

\section{Introduction}

Many cases of esophageal squamous cell carcinoma (SCC) are detected at the advanced stage of tumor progression [1] [2]. Most patients with advanced esophageal SCC have a poor outcome when treated with surgery alone. However, evidence from locoregional 
esophageal cancer indicates that treatment regimens that combined neoadjuvant chemoradiotherapy (CRT) with surgical resection are associated with improved patient outcomes [3] [4] [5] [6]. Preoperative CRT has been introduced in an attempt to increase the rates of complete resection by downsizing the primary tumor, and beyond that with the goal of improving local tumor control and preventing the formation of distant metastases [7]. However, perioperative mortality and morbidity are frequent in esophageal cancer patients. On the other hand, some patients whose tumors show a good response to neoadjuvant therapy may be cured without undergoing surgery. Therefore, it is important to establish a method with the ability to predict which patients will have a partial versus those who will have a complete response. Anatomical imaging methodologies, including computed tomography (CT), magnetic resonance imaging (MRI), and endoscopic ultrasound (EUS) are frequently used to determine tumor size and thus provide some insight into a patients response to therapy [8] [9] [10] [11]. When conventional imaging methods are used to assess a patient's response to neoadjuvant therapy, it is not possible to differentiate reliably between tumor cells and inflammatory reactions, edema and scar tissue [8]. The role and potential value of positron emission tomography (PET) scanning for monitoring certain tumors have been widely investigated in recent years [12] [13] [14]. In particular, PET conjugated with 18-F-fluorodeoxyglucose (FDG) provides physiological information that enables cancers to be diagnosed on the basis of altered tissue metabolism [15] [16]. Markedly increased FDG uptake in esophageal SCC has been documented in several studies [17] [18] [19]. Generally, the relationship between a pathological response and FDG-PET imaging is discussed on the basis of a decreased rate of standardized uptake value (SUV). However, with this methodology, it is difficult to discriminate between patients with the potential for a pathologically partial versus a complete response. Therefore, in this study, we retrospectively assessed the absolute SUV of FDG-PET in comparison with the decreased rate of SUV to evaluate the response of advanced esophageal SCC to neoadjuvant CRT, and their correlation with the pathological findings.

\section{Patients and Methods}

\subsection{Patients}

Patients with histologically confirmed primary ESCC were eligible for this study. The inclusion criteria were a performance status less than 2 , white blood cells $>3000 / \mu \mathrm{L}$, platelets $>100,000 / \mu \mathrm{L}$, serum total bilirubin $<2.0 \mathrm{mg} / \mathrm{dL}$, serum transaminase $<3$ times the upper normal limit, serum creatinine $<1.5 \mathrm{mg} / \mathrm{dL}$, creatinine clearance $>60$ $\mathrm{mL} / \mathrm{min}$ and no prior chemotherapy or radiotherapy. The exclusion criteria included concomitant malignancies, heart disease and patients with an esophago-bronchial fistula. Between June 1999 and December 2007, 65 patients with advanced and unresectable thoracic esophageal SCCs were enrolled into this study at the Department of General Surgical Science, Gunma University Graduate School of Medicine. Patients who had carcinomas at T3 or more advanced stages, according to the TNM classification of the UICC ( $6^{\text {th }}$ edition), on radiologic examinations were included. Thirty-two of the 65 pa- 
tients were considered to have inoperable tumors due to distant organ metastasis, distant lymph node metastasis, severe organ dysfunction and rejection of surgery by the patients. The remaining 33 (27 men and 6 women) were assigned to neoadjuvant treatment followed by surgery (Table 1 ). None of the patients had received prior treatment. The median age was 64.2 years, with a range of 42 to 77 years. The tumor stage and disease grade were classified according to the TNM classification (sixth edition) of the International Union Against Cancer (UICC). The tumor stage was determined conventionally by neck, chest and abdominal CT, bone scans, EUS, endoscopy and esophagography. Patients with locally advanced esophageal SCCs without distant organ metastasis were included. All of the sites of distant metastases were lymph nodes (e.g. cervical, supraclavicular or celiac Lymph nodes which are treated as regional lymph nodes in the classification of the Japan Esophageal Society [20]). None of the patients had diabetes, and in all patients blood sugar levels were less than $110 \mathrm{mg} / \mathrm{dL}$ at the time of the PET scan.

Table 1. The clinicopathologic characteristics of patients.

\begin{tabular}{|c|c|c|c|}
\hline Parameters & $5-\mathrm{FU} / \mathrm{CDGP}+\mathrm{RT}(\mathrm{n}=17)$ & $\mathrm{DOC}+\mathrm{RT}(\mathrm{n}=16)$ & $p$-value \\
\hline Age (mean \pm SD) & $63.3 \pm 12.5$ & $65.2 \pm 5.9$ & 0.59 \\
\hline Gender & & & 0.32 \\
\hline Male & 15 & 12 & \\
\hline Female & 2 & 4 & \\
\hline Location & & & 0.77 \\
\hline Ut & 3 & 3 & \\
\hline Mt & 9 & 10 & \\
\hline Lt & 5 & 3 & \\
\hline Tumor depth & & & 0.22 \\
\hline 3 & 4 & 6 & \\
\hline 4 & 13 & 10 & \\
\hline Lymph node metastasis & & & 0.91 \\
\hline 0 & 5 & 5 & \\
\hline 1 & 12 & 11 & \\
\hline Distant metastasis & & & 0.13 \\
\hline 0 & 16 & 13 & \\
\hline 1 & 1 & 3 & \\
\hline Stage & & & 0.38 \\
\hline 2 & 1 & 2 & \\
\hline 3 & 15 & 11 & \\
\hline 4 & 1 & 3 & \\
\hline
\end{tabular}

5-FU: 5-fluorouracil, CDGP: nedaplatin, DOC: docetaxel, RT: radiotherapy, SD: standard deviation, Ut: upper thoracic esophagus, Mt: middle thoracic esophagus, Lt: lower thoracic esophagus. 


\subsection{Neoadjuvant Treatment and Surgery}

After the diagnostic procedures, the patients underwent neoadjuvant treatment for 4 weeks, consisting of concurrent radiotherapy and chemotherapy. Tumor response was assessed by CT, endoscopy, esophagography and FDG-PET 2 weeks after the end of the treatment. The external radiotherapy was delivered by a two-field technique using a 10to $15-\mathrm{MV}$ photon beam at 2 Gy per fraction per day, 5 fractions per week, to a total of $40 \mathrm{~Gy}$. The concurrent chemotherapies used two type regimens (Table 1). Seventeen patients underwent chemotherapy that consisted of $80 \mathrm{mg} / \mathrm{m}^{2}$ nedaplatin administered intravenously over 3 hours on day 1 , and $350 \mathrm{mg} / \mathrm{m}^{2} 5$-flurouracil administered as a continuous intravenous infusion on days 1 through 5 . The other 16 patients received concurrent hyperthermo-chemotherapy, which consisted of $7 \mathrm{mg} / \mathrm{m}^{2}$ docetaxel administered intravenously for 1 hour before radiotherapy on days 1, 8, 15, and 22. External microwave hyperthermia was performed for 1 hour every week simultaneously with the chemotherapy using Thermotron RF-8 (Yamamoto Vinita, Osaka, Japan). All of the 33 patients underwent esophagectomy and regional lymph node dissection 4 weeks after the neoadjuvant treatment. Microscopic $0.5-\mathrm{cm}$ wide sections of the whole resected esophagus and stomach were prepared, and then fixed, embedded and stained with hematoxylin and eosin. Two pathologists, who were unaware of the patients' clinical responses, then reviewed the histopathology of each of the cases.

\subsection{PET Imaging}

The PET images were obtained using a SET 2400W (Shimadzu Corporation, Kyoto, Japan) with a $59.5-\mathrm{cm}$ transaxial field of view and a $20-\mathrm{cm}$ axial field of view. This produced 63 image planes spaced $3.125 \mathrm{~mm}$ apart. Transaxial spatial resolution was 4.2 $\mathrm{mm}$ FWHM at the center of the field of view and axial resolution was $5.0 \mathrm{~mm}$ FWHM. A whole body image, using the simultaneous emission transmission method with a rotating external source, was initiated 40 minutes after injection of 275 - $370 \mathrm{MBq}$ FDG by the multiple-bed position technique. Four to five sections from head to thigh were imaged for 8 minute per section. Patients fasted for at least 4 hours before FDG-PET scanning. The FDG-PET imaging protocols were approved by the Institutional Review Board of our institute [21] and all patients gave informed consent before undergoing the examination.

Attenuation-corrected transaxial images were reconstructed by the ordered subsets expectation maximization (OS-EM) algorithm into $128 \times 128$ matrices with pixel dimensions of $4.0 \mathrm{~mm}$ in-plane and $3.125 \mathrm{~mm}$ axially. Finally, every three consecutive slices were summated to generate a transaxial image $9.8 \mathrm{~mm}$ thick. This was used for visual interpretation and quantitative analysis. Similarly, coronal images $9.8 \mathrm{~mm}$ thick were also reconstructed from attenuation-corrected transaxial images. All PET images were evaluated qualitatively by at least two experienced nuclear medicine physicians. Functional images of SUV were also produced using the attenuation-corrected transaxial images, the amount of injected FDG, body weight, and the cross-calibration factors between PET and the dose calibrator. Thus SUV was defined as: 
$\mathrm{SUV}=$ the radioactive concentration in the tissue or lesion $(\mathrm{MBq} / \mathrm{g}) /$ injected dose $(\mathrm{MBq}) /$ patient's body weight $(\mathrm{g})$

Regions of interest (ROIs), consisting of areas $1 \mathrm{~cm}$ in diameter including the maximum uptake value, were drawn on the area corresponding to lesions exceeding $2 \mathrm{~cm}$ in diameter. If the lesion was $2 \mathrm{~cm}$ or smaller in diameter, the ROI was drawn over the entire lesion but the partial volume effect was not corrected. For primary lesions that were not visualized on PET imaging, the ROIs were drawn over the corresponding area using a fusion image technique combined with the CT and MRI images. Similarly, for affected regional lymph nodes that were not visualized on PET imaging, ROIs $0.6 \mathrm{~cm}$ in diameter were drawn on the corresponding area using the fusion image combined with CT images. A background ROI (with the same diameter as that of the lesion-based ROI) was drawn over the corresponding opposite area. If the lesion was located near the center of the body (as with the primary esophageal cancer) the background ROI was taken from a surrounding background area. The average value per pixel in the ROI used to assess the SUV was employed for semi-quantitative analysis.

PET scans were performed before and then two weeks after neoadjuvant therapy, and before the surgery.

\subsection{Response Evaluation}

Two weeks after the completion of treatment, the clinical response of each primary tumor was evaluated according to the criteria of the Japan Esophageal Society (JES) [22]. The assessment involved repeat EGD and CT, whereby two investigators conducted EGD, who examined the macroscopic findings of each tumor before and after treatment. All patients underwent a CT scan of the neck, chest and abdomen. Ten millimeter continuous scans were obtained from the neck to the bottom of the liver after intravenous injection of contrast medium. The endoscopic and CT results were discussed by the investigators, and the responses of the tumors were classified as follows. Target lesions (measurable lesions detected at baseline. A maximum of five lesions per organ should be identified as "target lesion"): complete response (CR), defined as the complete disappearance of all clinical evidence of existing lesions; partial response (PR), defined as a decrease in the size of the target lesion of more than $30 \%$; progressive disease (PD), defined as an increase in target lesion size of more than 20\%; or stable disease (SD), defined as an increase in tumor size of less than $20 \%$ or a decrease in target lesion size of less than $30 \%$. Non-target lesions (any other lesions including primary site of esophagus): complete response (CR), defined as the disappearance of all non-target lesions. In addition, primary lesion must disappear on endoscopy; incomplete response/ stable disease (IR/SD), defined as the persistence of one or more non-target lesion(s). In addition, the response of primary lesion is judged as IR/SD when its response does not meet the conditions for complete response or progressive disease on endoscopy; progressive disease (PD), defined as the appearance of one or more new lesions and/or unequivocal progression in existing non-target lesion(s). In addition, the primary lesion shows distinct tumor growth or progression in esophageal stenosis compared with the 
best condition during treatment. Overall response was classified according to the combinations of tumor response in target and non-target lesions with or without the appearance of new lesions (Table 2). The histopathological response to treatment was classified as grade $0,1,2$ or 3 in accordance with the criteria of the JES. Briefly, the degree of viability of the residual tumor cells was assessed as follows: Grade 3, defined as histological fibrosis with or without inflammation extending through the different layers of the esophageal wall, but no viable residual tumor cells; Grade 2, defined as less than one third of the residual tumor cells were viable; Grade 1, defined as more than one third of the residual tumor cells were viable; Grade 0 , no change.

\section{Results}

According to the PET scans on the 33 patients, mean maximum SUV before neoadjuvant therapy was 8.09. At the end of neoadjuvant therapy, mean maximum SUV was 4.48 , but decreased to 2.58 before surgery (Figure 1). Meanwhile, the histopathological examination revealed that ten out of 33 samples were Grade 1, 15 were Grade 2, and 8 were Grade 3. There was no significant relationship between clinical effect and histopathological findings (Table 3).

The relationship between histopathological response and the decreased rate of the maximum SUV was significantly different between the two groups (Figure 2). Mean rates of the maximum SUV at the end of neoadjuvant therapy as they related to mean rates of the maximum SUV before neoadjuvant therapy were as follows: Grade 1, 66\%; Grade 2, 55\%; Grade 3, 37\%. Likewise, before surgery, mean rates of the maximum SUV were as follows: Grade 1, 42\%; Grade 2, 27\%; Grade 3, $20 \%$.

\subsection{The Relationship between Decrease Rate of the Maximum SUV and Histopathological Response}

The mean decreased rate of the maximum SUV from the initial PET scans to that before surgery was $65 \%$. Therefore, evaluation of the therapeutic effect was performed using this value (Table 4). The histopathological response was divided as follows:

Table 2. Overall response.

\begin{tabular}{cccc}
\hline Target lesions & Non-target lesions & New lesions & Overall response \\
\hline CR & CR & No & CR \\
CR & IR/SD & No & PR \\
PR & Non-PD & No & PR \\
SD & Non-PD & No & SD \\
SD & Any & Yes or No & PD \\
Any & PD & Yes or No & PD \\
Any & Any & Yes & PD \\
\hline
\end{tabular}

CR: complete response, IR/SD: incomplete response/stable disease, PR: partial response, PD: progressive disease, SD: stable disease. 


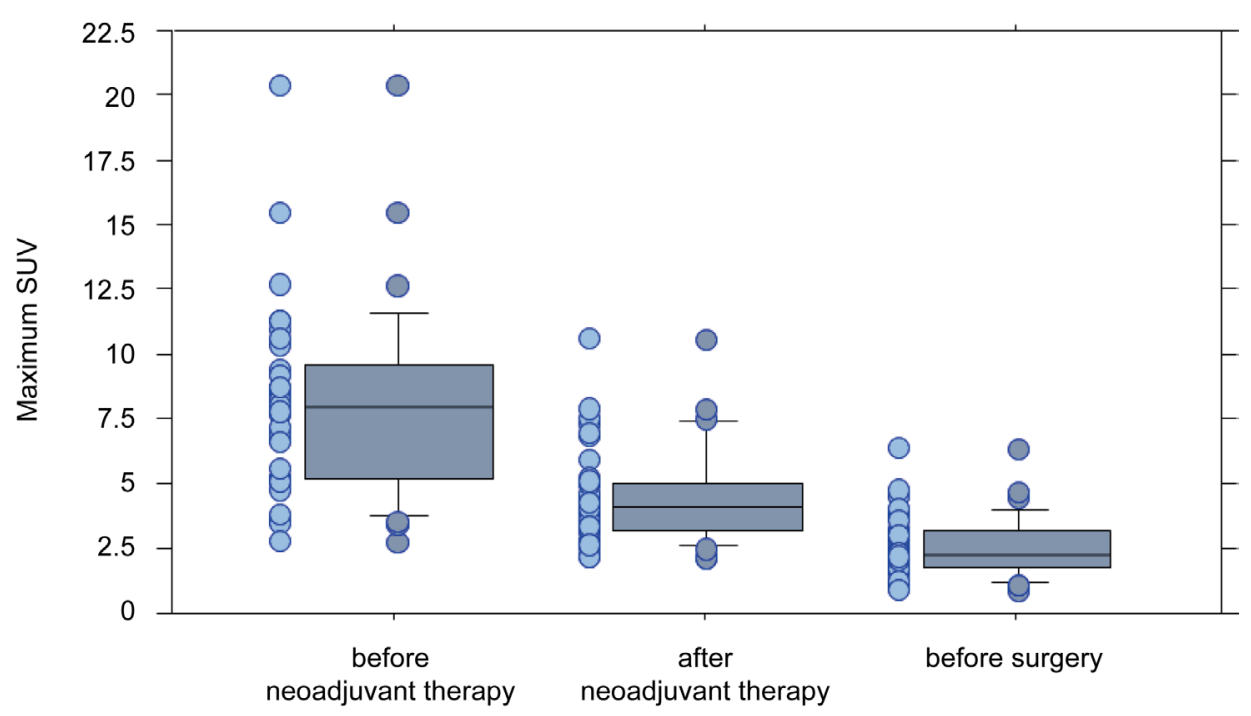

Figure 1. Mean maximum SUV was measured at three points. Analysis revealed that the mean maximum SUV before neoadjuvant therapy was 8.09. The maximum SUV at the end of neoadjuvant therapy was 4.48 , and 2.58 before surgery.

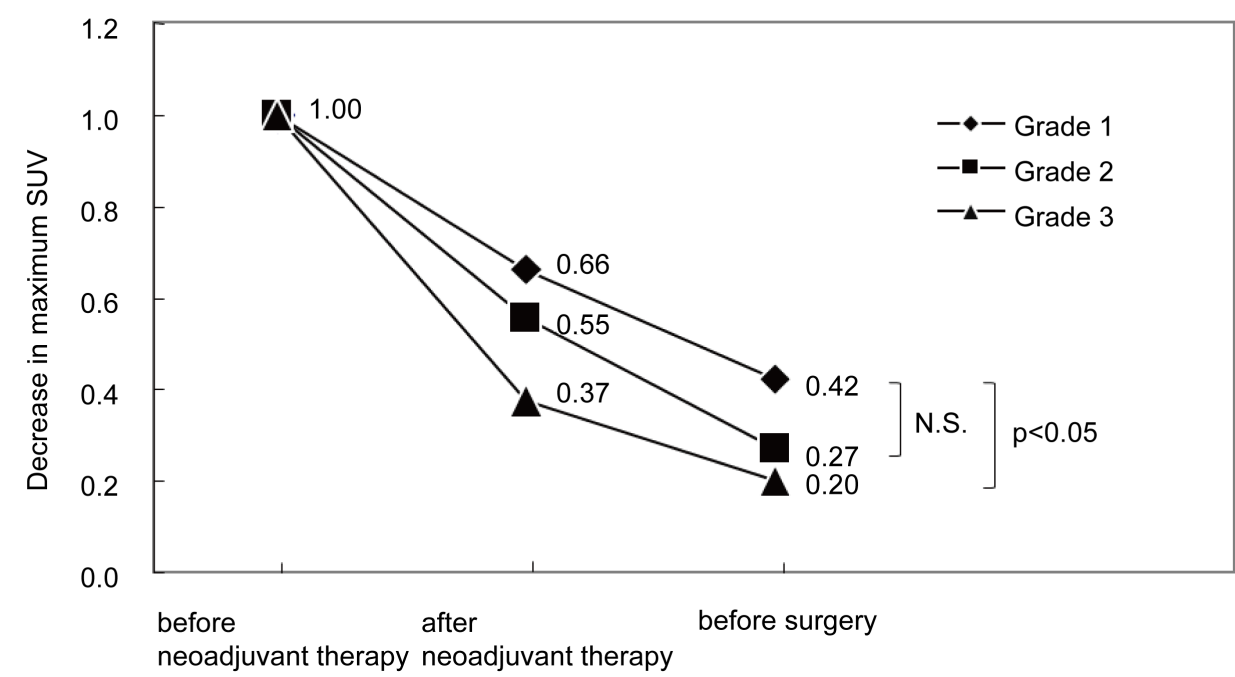

Figure 2. Relationship between the observed histopathological response and mean decreased rate of SUV. Mean rates of the maximum SUV at the end of neoadjuvant therapy were related to the values before neoadjuvant therapy as outlined below: Grade 1, 66\%; Grade 2, 55\%; Grade 3, 37\%. Similarly, mean rates of the maximum SUV at the end of neoadjuvant therapy relative those values before surgery were as follows: Grade 1, 46\%; Grade 2, 30\%; Grade 3, 20\%.

Table 3. The relationship clinical and pathological findings.

\begin{tabular}{ccccc}
\hline & \multicolumn{3}{c}{ Histological findings } & \\
\cline { 2 - 4 } & Grade 1 & Grade 2 & Grade 3 & 0.40 \\
\hline Clinical evaluation & 6 & 5 & 3 & \\
SD & 4 & 10 & 5 & \\
PR & & & \\
\hline
\end{tabular}

SD: stable disease, PR: partial response. 
Table 4. Evaluation of therapeutic effect according to decrease rate of maximum SUV.

\begin{tabular}{cccccc}
\hline PET $($ SUV $\geq 65 \%)$ & sensitivity & specificity & accuracy & PPV & NPV \\
\hline pCR/non-pCR & $5 / 8(62.5 \%)$ & $11 / 25(44.0 \%)$ & $16 / 33(48.5 \%)$ & $5 / 19(26.3 \%)$ & $11 / 14(78.6 \%)$ \\
Responder/non responder & $15 / 23(65.2 \%)$ & $6 / 10(60.0 \%)$ & $21 / 33(63.6 \%)$ & $15 / 19(78.9 \%)$ & $6 / 14(42.9 \%)$ \\
\hline
\end{tabular}

PPV: positive predictive value, NPV: negative predictive value; *: Grade 1 specimens were defined as non-responders, and Grade 2 and 3 were defined as responders.

Grade 3, pathological CR; Grade 1 and 2, pathological non-CR. At the same time, Grade 1 specimens were defined as non-responders, and Grades 2 and 3 were defined as responders. According to this definition, the sensitivity of distinguishing a pathological CR from non CR was $63 \%$. The specificity was $44 \%$, the accuracy was $48 \%$ and the positive predictive value (PPV) was $26 \%$. In contrast, the sensitivity, specificity, accuracy and PPV of distinguishing responders from non-responders were $65 \%, 60 \%, 64 \%$ and $79 \%$.

\subsection{The Relationship between Decrease Rate of the Maximum SUV and Histopathological Response}

Because the sensitivity, specificity and accuracy based on the decreased rate of the maximum SUV were so poor, the absolute maximum SUV was evaluated to distinguish between pathological CR or responders. Evaluation of therapeutic effect was performed using the maximum SUV 2.0 as the boundary line (Table 5, Figure 3). According to this system, the sensitivity of distinguishing between pathological CR from non-CR patients was $88 \%$. The specificity was $56 \%$, the accuracy was $64 \%$ and the PPV was $39 \%$. However, the sensitivity, specificity, accuracy, and PPV of distinguishing between responders from non-responders was $74 \%, 90 \%, 79 \%$ and $94 \%$, respectively.

\section{Discussion}

Despite many improvements in diagnosis and treatment, the 5-year survival rates after potentially curative esophagectomy rarely exceed $40 \%$ [23]. In 2007, a meta-analysis provided evidence that presented a compelling argument for neoadjuvant chemo (radio) therapy, followed by surgery, as the best strategy for ensuring long term survival. However, perioperative mortality and morbidity are high in esophageal cancer patients. Yet, some patients whose tumors show a good response to neoadjuvant therapy may be cured without undergoing surgery [24]. Therefore, it is important to establish a method that allows clinicians to predict a patient's likely response to treatment (i.e., a partial or pathologically complete responder). Although anatomical imaging procedures that include EGD, CT, MRI and EUS have been used to determine the response to therapy, up to until now, they have lacked the ability to differentiate between viable tumor cells and inflammatory reactions, edema and scar tissue. For this reason, FDG-PET is an imaging methodology with the potential to aid clinicians in predicting a patient's response to CRT. In our previous study, we reported that FDG-PET may be of value in assessing the pathological response to neoadjuvant therapy. In particular, low FDG uptake after 
Table 5. Evaluation of therapeutic effect according to absolute maximum SUV.

\begin{tabular}{cccccc}
\hline PET $($ SUV $<2)$ & sensitivity & specificity & accuracy & PPV & NPV \\
\hline pCR/non-pCR & $7 / 8(87.5 \%)$ & $14 / 25(56.0 \%)$ & $21 / 33(63.6 \%)$ & $7 / 18(38.9 \%)$ & $14 / 15(93.3 \%)$ \\
Responder/non responder & $17 / 23(73.9 \%)$ & $9 / 10(90.0 \%)$ & $26 / 33(78.8 \%)$ & $17 / 18(94.4 \%)$ & $9 / 15(60.0 \%)$ \\
\hline
\end{tabular}

PPV: positive predictive value, NPV: negative predictive value; *: Grade 1 specimens were defined as nonresponders, and Grade 2 and 3 were defined as responders.

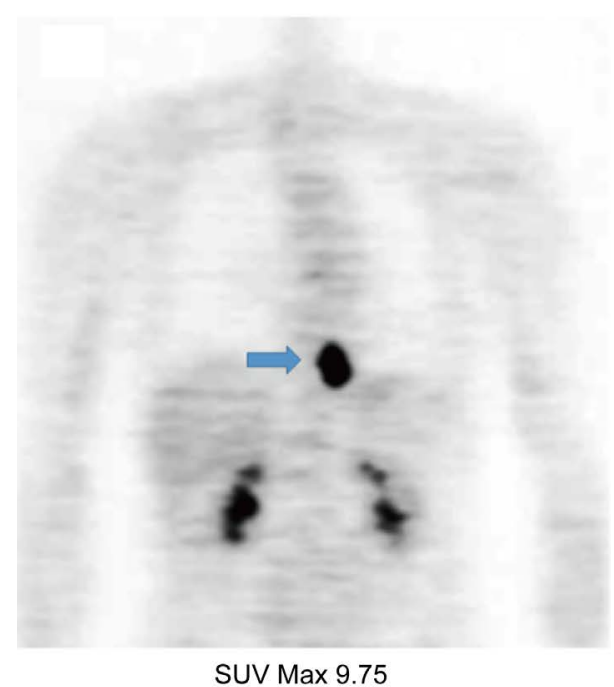

(a)

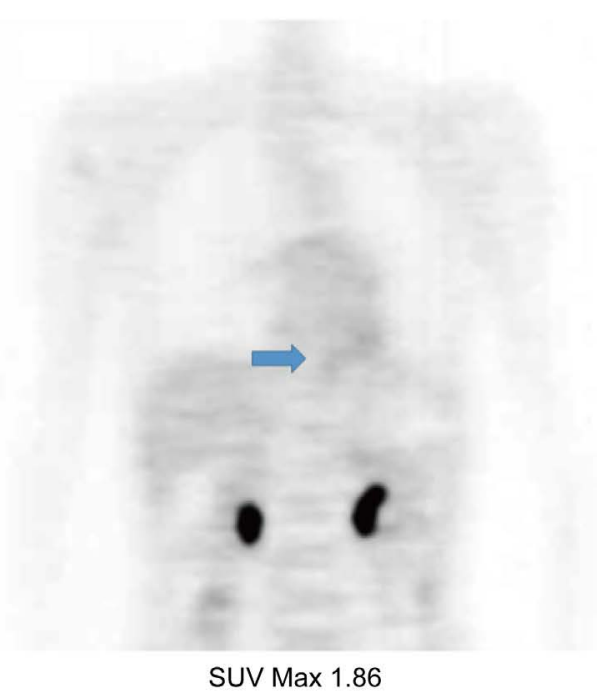

(b)

Figure 3. PET scan of a patient rated as having a pathological CR (Grade 3). The patient was 68 years old, male. He had a type 3 tumor in lower thoracic esophagus which was suspected to have invaded to the descending aorta. The patient underwent neoadjuvant hyperthermo-CRT using docetaxel. (a) The lesion demonstrated significant FDG uptake (arrow; the maximum SUV 9.75) and was detected prior to neoadjuvant therapy; (b) Three weeks following CRT, the FDG uptake was significantly less (arrow; the maximum SUV 1.86). The patient underwent esophagectomy a week after the second PET scan. Pathological examination of the removed specimen revealed no viable tumor cells.

therapy and a reduction in the extent of FDG uptake may provide a reliable assessment of a patient's response to therapy [25].

In this study, we assessed whether absolute maximum SUV or a decreased rate of the maximum SUV prior to CRT is a better predictor of a patients response to therapy. On the basis of our findings, the better indicator appeared to be absolute maximum SUV. In particular, absolute maximum SUV seemed to be a good predictor with the ability to distinguish responders from nonresponders, as well as a metric with the ability to predict pathological CRs. Previous studies have shown that FDG-PET is a modality with the ability predict responses to CRT. For instance, in rectal cancer, the reduction rate has been established as a good predictor of a pathological response through CRT [26].

In breast cancer, the reduction in SUV after chemotherapy has been identified as a solid predictor of pathologic responses [27]. Moreover, in esophageal cancer, the efficacy of FDG-PET as a predictor of patient responses to CRT has also been reported 
[28]. However, most of these studies discussed the efficacy on the basis of a reduction in SUV. By establishing the optimal reduction rate, although pathological or clinical responder may be distinguished from non-responders, it is difficult to identify pathological complete responders. Subtotal esophagectomy for advanced esophageal cancer is an invasive operation and mortality and morbidity rates are still high [29] [30] [31]. Therefore, the ability to distinguish tumors with the potential for a complete response through CRT is of great clinical value. However, a negative aspect of definitive CRT is that the incidence of local recurrence is higher than following curative surgery. The reason for local recurrence may be that a small number of viable tumor cells remained in those tumors evaluated as clinically complete responders. To avoid local recurrence, a method with the capacity to distinguish the cases that achieved a complete pathological response by definitive CRT are certainly desirable. The potential to identify a modality with the ability to identify pathological complete responders, means that unnecessary surgeries could be avoided.

The mean maximum SUV reduction rate of $65 \%$ revealed a lower sensitivity, specificity, accuracy and PPV than those seen when examining the actual maximum SUV. The SUV of FDG-PET itself reflects glucose metabolism. Therefore, reduction rates may be a good index of pathologic responses, but not the number of apoptotic cells. To begin to evaluate the number of residual viable tumor cells, the actual value of the maximum SUV should be evaluated. However, the number of examined patients was limited. Therefore, we will continue neoadjuvant treatment and surgery, and discuss the validity of this criterion prospectively.

\section{Conclusion}

In conclusion, to identify complete or good responders of neoadjuvant CRT for esophageal cancer, the absolute maximum SUV value is a better predictor than the decreased rate of the maximum SUV. We will continue neoadjuvant treatment and confirm the validity of this criterion.

\section{Conflict of Interest Statement}

Masanobu Nakajima and other co-authors have no conflict of interest.

\section{References}

[1] Kuwano, H., Nakajima, M., Miyazaki, T. and Kato, H. (2003) Distinctive Clinicopathological Characteristics in Esophageal Squamous Cell Carcinoma. Annals of Thoracic and Cardiovascular Surgery, 9, 6-13.

[2] Kato, H. (1995) Diagnosis and Treatment of Esophageal Neoplasms. Japanese Journal of Cancer Research, 86, 993-1009. https://doi.org/10.1111/j.1349-7006.1995.tb03012.x

[3] Campbell, N.P. and Villaflor, V.M. (2010) Neoadjuvant Treatment of Esophageal Cancer. World Journal of Gastroenterology, 16, 3793-3803. https://doi.org/10.3748/wjg.v16.i30.3793

[4] Courrech, Staal, E.F., Aleman, B.M., Boot, H., van Velthuysen, M.L., van Tinteren, H. and van Sandick, J.W. (2010) Systematic Review of the Benefits and Risks of Neoadjuvantchemoradiation for Oesophageal Cancer. British Journal of Surgery, 97, 1482-1496. 
https://doi.org/10.1002/bjs.7175

[5] Díaz, R., Reynes, G., Tormo, A., de Juan, M., Gironés, R., Segura, A., Aparicio, J., Richart, P., de la Cueva, H. and García, J. (2009) Long-Term Results of Neoadjuvant Chemotherapy and Combined Chemoradiotherapy before Surgery in the Management of Locally Advanced Oesophageal Cancer: A Single-Centre Experience. Clinical and Translational Oncology, 11, 835-841. https://doi.org/10.1007/s12094-009-0452-5

[6] Morgan, M.A., Lewis, W.G., Casbard, A., Roberts, S.A., Adams, R., Clark, G.W., Havard, T.J. and Crosby, T.D. (2009) Stage-for-Stage Comparison of Definitive Chemoradiotherapy, Surgery Alone and Neoadjuvant Chemotherapy for Oesophageal Carcinoma. British Journal of Surgery, 96, 1300-1307. https://doi.org/10.1002/bjs.6705

[7] Raoul, J.L., Le Prise, E., Meunier, B., Heresbach, D., Campion, J.P. and Launois, B. (1998) Neoadjuvant Chemotherapy and Hyperfractionated Radiotherapy with Concurrent LowDose Chemotherapy for Squamous Cell Esophageal Carcinoma. International Journal of Radiation Oncology ${ }^{\star}$ Biology ${ }^{\star}$ Physics, 42, 29-34. https://doi.org/10.1016/S0360-3016(98)00192-8

[8] Dittler, H.J., Fink, U. and Siewert, G.R. (1994) Response to Chemotherapy in Esophageal Cancer. Endoscopy, 26, 769-771. https://doi.org/10.1055/s-2007-1009101

[9] Laterza, E., de Manzoni, G., Guglielmi, A., Rodella, L., Tedesco, P. and Cordiano, C. (1999) Endoscopic Ultrasonography in the Staging of Esophageal Carcinoma after Preoperative Radiotherapy and Chemotherapy. The Annals of Thoracic Surgery, 67, 1466-1469. https://doi.org/10.1016/S0003-4975(99)00267-2

[10] Jones, D.R., Parker, L.A., Detterbeck, F.C. and Egan, T.M. (1999) Inadequacy of Computed Tomography in Assessing Patients with Esophageal Carcinoma after Induction Chemoradiotherapy. Cancer, 85, 1026-1032. https://doi.org/10.1002/(SICI)1097-0142(19990301)85:5<1026::AID-CNCR3>3.0.CO;2-N

[11] Griffith, J.F., Chan, A.C., Chow, L.T., Leung, S.F., Lam, Y.H., Liang, E.Y., Chung, S.C. and Metreweli, C. (1999) Assessing Chemotherapy Response of Squamous Cell Oesophageal Carcinoma with Spiral CT. The British Journal of Radiology, 72, 678-684. https://doi.org/10.1259/bjr.72.859.10624325

[12] Ahuja, V., Coleman, R.E., Herndon, J. and Patz, E.F. (1998) The Prognostic Significance of Fluorodeoxyglucose Positron Emission Tomography Imaging for Patients with Nonsmall Cell Lung Carcinoma. Cancer, 83, 918-924. https://doi.org/10.1002/(SICI)1097-0142(19980901)83:5<918::AID-CNCR17>3.0.CO;2-Y

[13] Higashi, K., Ueda, Y., Ayabe, K., Sakurai, A., Seki, H., Nambu, Y., Oguchi, M., Shikata, H., Taki, S., Tonami, H., Katsuda, S. and Yamamoto, I. (2000) FDG PET in the Evaluation of the Aggressiveness of Pulmonary Adenocarcinoma: Correlation with Histopathological Features. Nuclear Medicine Communications, 21, 707-714. https://doi.org/10.1097/00006231-200008000-00002

[14] Kato, H., Kuwano, H., Nakajima, M., Miyazaki, T., Yoshikawa, M., Ojima, H., Tsukada, K., Oriuchi, N., Inoue, T. and Endo, K. (2002) Comparison between Positron Emission Tomography and Computed Tomography in the Use of the Assessment of Esophageal Carcinoma. Cancer, 94, 921-928. https://doi.org/10.1002/cncr.10330

[15] Bar-Shalom, R., Valdivia, A.Y. and Blaufox, M.D. (2000) PET Imaging in Oncology. Seminars in Nuclear Medicine, 30, 150-185. https://doi.org/10.1053/snuc.2000.7439

[16] Kato, H., Takita, J., Miyazaki, T., Nakajima, M., Fukai, Y., Masuda, N., Fukuchi, M., Manda, R., Ojima, H., Tsukada, K., Kuwano, H., Oriuchi, N. and Endo, K. (2003) Correlation of 18-F-Fluorodeoxyglucose (FDG) Accumulation with Glucose Transporter (Glut-1) Expression in Esophageal Squamous Cell Carcinoma. Anticancer Research, 23, 3263-3272. 
[17] Rice, T.W. (2000) Clinical Staging of Esophageal Carcinoma. CT, EUS, and PET. Chest Surgery Clinics of North America, 10, 471-485.

[18] Kole, A.C., Plukker, J.T., Nieweg, O.E. and Vaalburg, W. (1998) Positron Emission Tomography for Staging of Oesophageal and Gastroesophageal Malignancy. British Journal of Cancer, 78, 521-527. https://doi.org/10.1038/bjc.1998.526

[19] Kato, H., Miyazaki, T., Nakajima, M., Fukuchi, M., Manda, R. and Kuwano, H. (2004) Value of Positron Emission Tomography in the Diagnosis of Recurrent Oesophageal Carcinoma. British Journal of Surgery, 91, 1004-1009. https://doi.org/10.1002/bjs.4595

[20] Japan Esophageal Society (2009) Japanese Classification of Esophageal Cancer, Tenth Edition: Part I. Esophagus, 6, 1-25. https://doi.org/10.1007/s10388-009-0169-0

[21] Inoue, T., Oriuchi, N., Kunio, M., Tomiyoshi, K., Tomaru, Y., Aoyagi, K., Amano, S., Suzuki, H., Aoki, J., Sato, T. and Endo, K. (1999) Accuracy of Standardized Uptake Value Measured by Simultaneous Emission and Transmission Scanning in PET Oncology. Nuclear Medicine Communications, 20, 849-857.

https://doi.org/10.1097/00006231-199909000-00012

[22] Japan Esophageal Society (2009) Japanese Classification of Esophageal Cancer, Tenth Edition: Part II and III. Esophagus, 6, 71-94. https://doi.org/10.1007/s10388-009-0193-0

[23] Ando, N., Iizuka, T., Kakegawa, T., Isono, K., Watanabe, H., Ide, H., Tanaka, O., Shinoda, M., Takiyama, W., Arimori, M., Ishida, K. and Tsugane, S. (1997) A Randomized Trial of Surgery with and without Chemotherapy for Localized Squamous Carcinoma of the Thoracic Esophagus: The Japan Clinical Oncology Group Study. The Journal of Thoracic and Cardiovascular Surgery, 114, 205-209. https://doi.org/10.1016/S0022-5223(97)70146-6

[24] Stahl, M., Stuschke, M., Lehmann, N., Meyer, H.J., Walz, M.K., Seeber, S., Klump, B., Budach, W., Teichmann, R., Schmitt, M., Schmitt, G., Franke, C. and Wilke, H. (2005) Chemoradiation with and without Surgery in Patients with Locally Advanced Squamous Cell Carcinoma of the Esophagus. Journal of Clinical Oncology, 23, 2310-2317. https://doi.org/10.1200/JCO.2005.00.034

[25] Kato, H., Kuwano, H., Nakajima, M., Miyazaki, T., Yoshikawa, M., Masuda, N., Fukuchi, M., Manda, R., Tsukada, K., Oriuchi, N. and Endo, K. (2002) Usefulness of Positron Emission Tomography for Assessing the Response of Neoadjuvant Chemoradiotherapy in $\mathrm{Pa}$ tients with Esophageal Cancer. The American Journal of Surgery, 184, 279-283.

https://doi.org/10.1016/S0002-9610(02)00932-7

[26] Kato, H., Fukuchi, M., Miyazaki, T., Nakajima, M., Tanaka, N., Inose, T., Kimura, H., Faried, A., Saito, K., Sohda, M., Fukai, Y., Masuda, N., Manda, R., Ojima, H., Tsukada, K., Oriuchi, N., Endo, K., Nonaka, T., Shioya, M., Ishikawa, H., Sakurai, H., Nakano, T. and Kuwano, H. (2007) Prediction of Response to Definitive Chemoradiotherapy in Esophageal Cancer Using Positron Emission Tomography. Anticancer Research, 27, 2627-2633.

[27] Leibold, T., Akhurst, T.J., Chessin, D.B., Yeung, H.W., Macapinlac, H., Shia, J., Minsky, B.D., Saltz, L.B., Riedel, E., Mazumdar, M., Paty, P.B., Weiser, M.R., Wong, W.D., Larson, S.M. and Guillem, J.G. (2011) Evaluation of (18)F-FDG-PET for Early Detection of Suboptimal Response of Rectal Cancer to Preoperative Chemoradiotherapy: A Prospective Analysis. Annals of Surgical Oncology, 18, 2783-2789. https://doi.org/10.1245/s10434-011-1634-2

[28] Dunnwald, L.K., Doot, R.K., Specht, J.M., Gralow, J.R., Ellis, G.K., Livingston, R.B., Linden, H.M., Gadi, V.K., Kurland, B.F., Schubert, E.K., Muzi, M. and Mankoff, D.A. (2011) PET Tumor Metabolism in Locally Advanced Breast Cancer Patients Undergoing Neoadjuvant Chemotherapy: Value of Static versus Kinetic Measures of Fluorodeoxyglucose Uptake. Clinical Cancer Research, 17, 2400-2409. https://doi.org/10.1158/1078-0432.CCR-10-2649

[29] van Heijl, M., Omloo, J.M., van Berge Henegouwen, M.I., Hoekstra, O.S., Boellaard, R., 
Bossuyt, P.M., Busch, O.R., Tilanus, H.W., Hulshof, M.C., van der Gaast, A., Nieuwenhuijzen, G.A., Bonenkamp, H.J., Plukker, J.T., Cuesta, M.A., Ten Kate, F.J., Pruim, J., van Dekken, H., Bergman, J.J., Sloof, G.W. and van Lanschot, J.J. (2011) Fluorodeoxyglucose Positron Emission Tomography for Evaluating Early Response during Neoadjuvant Chemoradiotherapy in Patients with Potentially Curable Esophageal Cancer. Annals of Surgery, 253, 56-63. https://doi.org/10.1097/SLA.0b013e3181f66596

[30] Avendano, C.E., Flume, P.A., Silvestri, G.A., King, L.B. and Reed, C.E. (2002) Pulmonary Complications after Esophagectomy. The Annals of Thoracic Surgery, 73, 922-926.

https://doi.org/10.1016/S0003-4975(01)03584-6

[31] Low, D.E. (2011) Update on Staging and Surgical Treatment Options for Esophageal Cancer. Journal of Gastrointestinal Surgery, 15, 719-729.

https://doi.org/10.1007/s11605-011-1515-9

Submit or recommend next manuscript to SCIRP and we will provide best service for you:

Accepting pre-submission inquiries through Email, Facebook, LinkedIn, Twitter, etc. A wide selection of journals (inclusive of 9 subjects, more than 200 journals)

Providing 24-hour high-quality service

User-friendly online submission system

Fair and swift peer-review system

Efficient typesetting and proofreading procedure

Display of the result of downloads and visits, as well as the number of cited articles

Maximum dissemination of your research work

Submit your manuscript at: http://papersubmission.scirp.org/

Or contact ojgas@scirp.org 